\title{
BMJ Open Protocol for a scoping review of outcomes in clinical studies of interventions for venous thromboembolism in adults
}

\author{
Tobias Tritschler (D) , ${ }^{1,2}$ Nicole Langlois, ${ }^{2}$ Brian Hutton (D) , ${ }^{2}$ Beverley J Shea, ${ }^{2}$ \\ Risa Shorr, ${ }^{2}$ Sara Ng, ${ }^{2}$ Suzanne Dubois, ${ }^{3}$ Carol West, ${ }^{3}$ Alfonso lorio, ${ }^{4}$ \\ Peter Tugwell, ${ }^{2}$ Grégoire Le Gal (D) ${ }^{2}$
}

To cite: Tritschler T, Langlois N, Hutton B, et al. Protocol for a scoping review of outcomes in clinical studies of interventions for venous thromboembolism in adults. BMJ Open 2020;10:e040122. doi:10.1136/ bmjopen-2020-040122

- Prepublication history and additional material for this paper is available online. To view these files, please visit the journal online (http://dx.doi.org/10. 1136/bmjopen-2020-040122)

Received 06 May 2020 Revised 26 0ctober 2020 Accepted 18 November 2020

\section{Check for updates}

(c) Author(s) (or their employer(s)) 2020. Re-use permitted under CC BY-NC. No commercial re-use. See rights and permissions. Published by BMJ.

${ }^{1}$ Department of General Internal Medicine, Inselspital, Bern University Hospital, University of Bern, Bern, Switzerland ${ }^{2}$ Department of Medicine, Ottawa Hospital Research Institute, University of Ottawa, Ottawa, Ontario, Canada

${ }^{3}$ Canadian Venous

Thromboembolism Research Network (CanVECTOR), Patient Partner Platform, Ottawa, Ontario, Canada

${ }^{4}$ Department of Medicine, McMaster University, Hamilton, Ontario, Canada

Correspondence to Dr Grégoire Le Gal; glegal@ohri.ca

\section{ABSTRACT}

Introduction Venous thromboembolism (VTE) is a common, potentially fatal yet treatable disease. Several advances in treatment of VTE have been made over the past decades, but definition and reporting of outcomes across those studies are inconsistent. Development of an international core outcome set for clinical studies of interventions for VTE addresses this lack of standardisation. The first step in the development of a core outcome set is to conduct a scoping review which aims to generate an inclusive list of unique outcomes that have been reported in previous studies.

Methods and analysis MEDLINE, Embase and the Cochrane Central Register of Controlled Trials will be searched with no language restriction for prospective studies reporting on interventions for treatment of VTE in patients who are adult and non-pregnant. Records will be sorted in reverse chronological order. Study screening and data extraction will be independently performed by two authors in blocks based on date of publication, starting with 2015 to 2020 and subsequent 1-year periods, until no new outcome measures are identified from the set of included studies. After homogenising spelling and combining outcomes with the same meaning, a list of unique outcomes will be determined. Those outcomes will be grouped into outcome domains. Qualitative analysis and descriptive statistics will be used to report results. Ethics and dissemination Ethical approval is not required for this study. The results of this scoping review will be presented at scientific conferences, published in a peer-reviewed journal, and they will provide candidate outcome domains to be considered in subsequent steps in the development of a core outcome set for clinical studies of interventions for VTE.

Protocol registration details http://hdl.handle.net/ 10393/40459

\section{INTRODUCTION}

\section{Rationale}

Venous thromboembolism (VTE) is a common condition manifested as deep vein thrombosis (DVT) or pulmonary embolism (PE). If left untreated, the reported mortality of $\mathrm{PE}$ has varied widely in historical and more recent studies, ${ }^{12}$ but timely and appropriate treatment with

\section{Strengths and limitations of this study}

The study will aim to provide a comprehensive review of all outcomes that have been reported in clinical studies of treatment for venous thromboembolism.

- The study will use best practice methods to conduct a scoping review.

- Randomised controlled trials and prospective cohort studies will be eligible.

- We will determine a list of unique outcomes and group those outcomes into domains.

- We will involve patient partners throughout the study planning, conduct and dissemination.

anticoagulant medications dramatically reduces fatal events to less than $1 \% .^{3}$ In addition to PE-related death, most VTE treatment studies incorporate recurrent VTE or bleeding as primary or secondary outcomes. However, it has been noted that the list, definition and reporting of outcomes is inconsistent across studies and discrepancies in how these outcomes are defined and measured have led to important challenges in comparing and synthesising the results of trials. ${ }^{4}$ Lack of valid and standardised definitions of domains and measures for certain outcomes has also compromised the ability to demonstrate clinically meaningful effects. For example, how severe a bleeding event should be to count as an outcome has been a matter of debate. ${ }^{5}$ The definition of PE-related death is variable between studies, ${ }^{6}$ and within a single study the outcome adjudicators demonstrated poor reproducibility (50\% discordant results) for unexplained sudden death adjudicated as PE-related. ${ }^{7}$ Beneficial and safety outcomes beyond mortality, recurrent VTE and bleeding, such as post-thrombotic syndrome, quality of life, symptom resolution or psychological effects, are rarely measured in VTE studies, ${ }^{89}$ even though their impact on patients' health and 
economic burden of VTE may be relevant. Of note, no study to date has assessed which outcomes are most important to patients and caregivers.

The development of a core outcome set (COS), defined as an agreed minimum set of outcomes that should be measured and reported in all trials of a specific condition, addresses this lack of standardisation. The Core Outcome Measures in Effectiveness Trials (COMET) and Outcome Measures in Rheumatology (OMERACT) initiatives aim to stimulate the development and application of COS, adopting best practices and robust methodology that are based on evidence. ${ }^{10}{ }^{11}$ The first step in the development of a COS includes a scoping review of the literature to identify outcomes and domains that have been used previously. The results of the scoping review will help to determine the heterogeneity of reported outcomes and establish a list of unique outcomes. The results of this scoping review, considered together with the findings of other planned qualitative work involving multiple stakeholders (including patients, caregivers, clinicians, researchers, clinical practice guideline developers, health technology assessors, payers, policymakers, public research funding agencies and drug developers), will inform candidate outcomes and domains for the final consensus process to determine the COS for clinical studies of interventions for VTE.

\section{Objectives}

Primary objective

The primary objective of the scoping review is to generate an inclusive list of unique outcome domains that have been reported in previous VTE treatment studies.

\section{Secondary objectives}

The secondary objectives are to assess the number of unique outcome domains and measures reported (ie, outcome reporting heterogeneity), ${ }^{12}$ to assess the number of unique outcomes reported per study, to assess the presence of different wording for the same outcomes, to assess different time points at which unique outcomes are measured, to assess the number of patient-reported unique outcomes and to capture outcome definitions.

\section{METHODS AND ANALYSIS}

This protocol was developed following guidance in the COMET and OMERACT handbooks and reporting adheres to the Preferred Reporting Items for Systematic Review and Meta-Analysis Protocols (PRISMA-P) statement. ${ }^{101113}$ The protocol of this scoping review has been registered with the University of Ottawa's digital repository of research (available at http://hdl.handle.net/ $10393 / 40459)$ and the VTE-COS project is registered with the COMET database (http://www.comet-initiative. org). Reporting of the scoping review will adhere to the PRISMA statement items on scoping reviews. ${ }^{14}$

\section{Eligibility criteria}

Participants

We will seek studies that enrolled adults diagnosed with VTE, either DVT of the legs, PE or both. We will exclude VTE studies which included only pregnant women, comprising the antepartum, peripartum and postpartum periods, because an ongoing systematic review already assesses reported outcomes in this specific population (PROSPERO registration number CRD42019111479).

\section{Interventions}

We are interested in nine categories of interventions for treatment of VTE: (1) anticoagulation; (2) aspirin and other non-steroidal anti-inflammatory drugs; (3) statins; (4) thrombolysis; (5) surgery; (6) venous filters; (7) pharmacomechanical catheter-directed thrombolysis; (8) venous angioplasty and stenting; and (9) compression stockings. Studies involving the evaluation of other therapies will be excluded. Because of the wide range of treatment options for VTE, some outcome domains may only be relevant to certain therapies or subpopulation of patients. While these may not be mandatory domains in the final core outcome set, they may represent important but optional domains or research agenda domains according to the OMERACT onion schema. ${ }^{11}$ As such, the categorisation of interventions will not only be collected to characterise included studies but also considered in study selection and data extraction (see 'data management and selection process').

\section{Outcome}

This review will capture all outcomes reported in the included studies. Each component of a composite outcome will be considered as single outcome.

\section{Study design}

Randomised controlled trials and prospective cohort studies will be eligible.

\section{Search strategy}

An experienced information specialist developed the strategy for structured database searches in consultation with the review team. The search strategy was peer reviewed according to the Peer Review of Electronic Search Strategies (PRESS) guideline statement by a second independent information specialist before being finalised. ${ }^{15}$ Databases to be searched will include MEDLINE, Embase and the Cochrane Central Register of Controlled Trials. No language restrictions will be in place. The search strategy for MEDLINE, Embase and the Cochrane Central Register of Controlled Trials for the first blocks of studies to be screened (ie, 2014 and 2015 to 2020; see 'data management and selection process') is provided in the online supplemental appendix.

\section{Data management and selection process}

Screening of studies at both the title/abstract level (Level 1 screening) and the full-text level (Level 2 screening) will be implemented in Covidence, an online software 
management programme for the performance of systematic reviews. ${ }^{16}$ All documents will be reviewed independently by two team members. Disagreement will be resolved by discussion or by involving a third reviewer, if needed. A flow diagram summarising the process of study selection will be prepared.

To maximise efficiency of the study selection process, titles/abstracts for Level 1 screening will be sorted in reverse chronological order and will be screened in blocks based on date of publication. To begin, studies published between 2015 and 2020 will be screened at both abstract and full-text level to establish a full set of included studies for this time period. Data extraction from these studies (methods described below) will be performed in full to establish a complete 'map' of all the outcomes measured in the set of included studies. Subsequently, in 1-year intervals (ie, 2014, 2013, 2012 and so on), the same approach will be taken; selection of additional studies for inclusion will be halted when, for a 1-year period, no new outcome measures are identified from the set of included studies (commonly referred to as 'saturation' in terms of outcome measures). This approach is recommended by COMET and OMERACT guidance and results in a robust source of information for the review while offering efficiencies in both study selection and data extraction. ${ }^{10} 11$ Because studies assessing different categories of interventions may be variably represented over time, saturation will be assessed for each of the nine above-defined intervention categories. If a certain category is not represented by at least one study within one of the assessment periods (ie, 2015 to 2020 and subsequent 1-year periods), selection of additional studies will not be halted for this particular category.

\section{Data extraction}

For all included studies identified using the above approach, extracted data from each report will include the characteristics of the study (design, year, country, interventions, follow-up duration), as well as the set of reported outcomes, outcome definitions, outcome measurement tools, timing of measurement and metrics. Outcome descriptions will be extracted verbatim. All data of interest will be extracted from the source publications by two authors independently. Corresponding authors of selected studies will be contacted for clarification on details of the reported outcomes, if needed.

\section{Outcomes and prioritisation}

The primary outcome of the study is a list of unique outcomes reported in the included studies. A unique outcome will be defined as one that has original meaning and context. ${ }^{12}$ Outcomes differing only in timing of the outcome assessment (eg, 10-day vs 30-day all-cause mortality) will not be considered unique. We will extract outcomes verbatim and also record each outcome's definition, timing of assessment, measurement and metrics. The selection process of unique outcomes will follow the proposal of Young et al. ${ }^{12}$ First, duplicate verbatim outcomes will be removed after homogenising spelling (eg, bleeding and bleed, rates and rate and so on). Second, outcomes meaning the same will be rewritten by two reviewers independently to develop non-verbatim outcomes. The rewriting process will be documented. Finally, outcomes remaining after removal of duplicate non-verbatim outcomes will define the list of unique outcomes.

\section{Risk of bias (quality) assessment}

Given that the purpose of this review is to develop an inclusive set of outcome domains in order to inform subsequent steps in the VTE-COS development, no risk of bias assessment will be performed. This is consistent with recommendations in the PRISMA extension for scoping reviews. ${ }^{14}$

\section{Data synthesis}

We will use descriptive statistics to report the number of verbatim outcomes reported overall and in each individual study and the number of terms used to describe a single unique outcome. Furthermore, we will report the number of unique outcomes overall and per study, definitions of unique outcomes including time points of assessment, as well as the number of physician-reported and patient-reported unique outcomes.

Similar unique outcomes will be grouped into domains. A domain is defined as a component or concept of 'an aspect of health or health condition that needs to be measured to appropriately assess the effects of a health intervention. ${ }^{11}$ Once several domains have been identified, outcomes within domains will be checked for internal homogeneity (ie, coherence of outcomes within domain) and external heterogeneity (ie, distinction to outcomes in other domains). ${ }^{17}$ As per OMERACT filter 2.1, the final list of domains will be categorised into four core areas including manifestations/abnormalities, life impact, death/lifespan and societal/resource use. ${ }^{18}$

We will use descriptive statistics to report the number of domains and core areas covered in each individual study and vice versa. In subgroup analyses, we will assess differences related to above-mentioned descriptive statistics between randomised controlled trials and prospective cohort studies, and between different locations of initial VTE (ie, studies for which patients were eligible if they had DVT vs PE vs VTE).

\section{Confidence in cumulative evidence}

Because we aim to capture and describe what outcomes have been reported in previous VTE studies, determination of the strength of evidence is not applicable.

\section{Patient and public involvement}

Patient partners of the Canadian Venous Thromboembolism Clinical Trials and Outcomes Research (CanVECTOR) Network are members of the Steering Committee of the VTE-COS project. As persons with lived experience or relatives thereof, they provided insights about patient-relevant aspects of the project and 
contributed to the concept and protocol of this scoping review. They will review the outcome domains and the final manuscript, and will be involved in the dissemination of the results by providing assistance in writing lay evidence summaries which will also serve as basis for discussion in subsequent steps of the COS development.

\section{ETHICS AND DISSEMINATION}

Ethical approval is not required for this study because this is a scoping review of published studies.

In this scoping review, we aim to generate a list of unique outcome domains reported in previous studies of VTE treatment. This list will serve to define core domains to be considered in the development of a COS for VTE treatment studies. Subsequent steps of the COS development project include (1) individual interviews and focus groups of different stakeholders to identify additional candidate domains which are important for patients and other stakeholders and to increase understanding of stakeholder-relevant outcomes; (2) a Delphi survey to add additional previously not identified outcomes and to prioritise outcomes; (3) a consensus meeting among the study researchers and key stakeholders to define the final COS; and (4) the dissemination of the final COS.

The findings of this scoping review will be disseminated through a peer-reviewed journal publication and presentations at scientific conferences. As part of the VTECOS project, the study is endorsed by the International Society on Thrombosis and Haemostasis (ISTH), the CanVECTOR network and the International Network of VENous Thromboembolism Clinical Research Networks (INVENT). In addition to publication and presentation of the study findings, we will use their platforms to foster dissemination of results to researchers, knowledge users and patients in form of audience-specific summary reports.

Acknowledgements Tobias Tritschler holds an Early Postdoc.Mobility Award from the Swiss National Science Foundation (SNSF P2ZHP3_177999) and a Fellowship Award from the CanVECTOR Network. Grégoire Le Gal holds an Early Researcher Award from the Province of Ontario, a mid-career clinician scientist award from the Heart and Stroke Foundation of Ontario and the Chair on the Diagnosis of Venous Thromboembolism, Department of Medicine, University of Ottawa.

Contributors TT, NL, BH, BS, RS, SN, SD, CW, Al, PT and GLG contributed to concept and design of the study. TT, NL, BH and GLG wrote the manuscript. TT, $\mathrm{NL}, \mathrm{BH}, \mathrm{BS}, \mathrm{RS}, \mathrm{SN}, \mathrm{SD}, \mathrm{CW}, \mathrm{Al}, \mathrm{PT}$ and GLG reviewed and revised the manuscript. $\mathrm{TT}, \mathrm{NL}, \mathrm{BH}, \mathrm{BS}, \mathrm{RS}, \mathrm{SN}, \mathrm{SD}, \mathrm{CW}, \mathrm{Al}, \mathrm{PT}$ and GLG approved the final version of the manuscript.

Funding The VTE-COS project is funded by the Canadian Institutes of Health Research (PJT-165897) and is supported by the CanVECTOR Network; the Network receives grant funding from the Canadian Institutes of Health Research (CDT142654). The funder played no role in developing the protocol.

Competing interests None declared.

Patient consent for publication Not required.

Provenance and peer review Not commissioned; externally peer reviewed.

Supplemental material This content has been supplied by the author(s). It has not been vetted by BMJ Publishing Group Limited (BMJ) and may not have been peer-reviewed. Any opinions or recommendations discussed are solely those of the author(s) and are not endorsed by BMJ. BMJ disclaims all liability and responsibility arising from any reliance placed on the content. Where the content includes any translated material, BMJ does not warrant the accuracy and reliability of the translations (including but not limited to local regulations, clinical guidelines, terminology, drug names and drug dosages), and is not responsible for any error and/or omissions arising from translation and adaptation or otherwise.

Open access This is an open access article distributed in accordance with the Creative Commons Attribution Non Commercial (CC BY-NC 4.0) license, which permits others to distribute, remix, adapt, build upon this work non-commercially, and license their derivative works on different terms, provided the original work is properly cited, appropriate credit is given, any changes made indicated, and the use is non-commercial. See: http://creativecommons.org/licenses/by-nc/4.0/.

\section{ORCID iDs}

Tobias Tritschler http://orcid.org/0000-0002-8775-0511

Brian Hutton http://orcid.org/0000-0001-5662-8647

Grégoire Le Gal http://orcid.org/0000-0002-9253-248X

\section{REFERENCES}

1 Nieto JA, Vicente JA, Prieto LM, et al. Thirty-day outcomes in patients with acute pulmonary embolism who discontinued anticoagulant therapy before 90 days. Am Heart J 2018;206:1-10.

2 Barritt DW, Jordan SC. Anticoagulant drugs in the treatment of pulmonary embolism. A controlled trial. Lancet 1960;1:1309-12.

3 Carrier M, Le Gal G, Wells PS, et al. Systematic review: case-fatality rates of recurrent venous thromboembolism and major bleeding events among patients treated for venous thromboembolism. Ann Intern Med 2010;152:578-89.

4 Witt DM, Nieuwlaat R, Clark NP, et al. American Society of hematology 2018 guidelines for management of venous thromboembolism: optimal management of anticoagulation therapy. Blood Adv 2018;2:3257-91.

5 Schulman S, Kearon C. Subcommittee on control of anticoagulation of the scientific standardization Committee of the International Society on thrombosis haemostasis. Definition of major bleeding in clinical investigations of antihemostatic medicinal products in nonsurgical patients. J Thromb Haemost 2005;3:692-4.

6 Kraaijpoel N, Tritschler T, Guillo E, et al. Definitions, adjudication, and reporting of pulmonary embolism-related death in clinical studies: a systematic review. J Thromb Haemost 2019;17:1590-607.

7 Girard P, Penaloza A, Parent F, et al. Reproducibility of clinical events adjudications in a trial of venous thromboembolism prevention. $J$ Thromb Haemost 2017;15:662-9.

8 Klok FA, Barco S, Siegerink B. Measuring functional limitations after venous thromboembolism: a call to action. Thromb Res 2019;178:59-62.

9 Kahn SR, Houweling AH, Granton J, et al. Long-Term outcomes after pulmonary embolism: current knowledge and future research. Blood Coagul Fibrinolysis 2014;25:407-15.

10 Williamson PR, Altman DG, Bagley H, et al. The comet Handbook: version 1.0. Trials 2017;18:280.

11 OMERACT Initiative. The OMERACT Handbook, 2018. Available: https://omeracthandbook.org [Accessed 21 Apr 2020].

12 Young AE, Brookes ST, Avery KNL, et al. A systematic review of core outcome set development studies demonstrates difficulties in defining unique outcomes. J Clin Epidemiol 2019;115:14-24.

13 Moher D, Shamseer L, Clarke M, et al. Preferred reporting items for systematic review and meta-analysis protocols (PRISMA-P) 2015 statement. Syst Rev 2015;4:1.

14 Tricco AC, Lillie E, Zarin W, et al. PRISMA extension for scoping reviews (PRISMA-ScR): checklist and explanation. Ann Intern Med 2018;169:467-73.

15 McGowan J, Sampson M, Salzwedel DM, et al. PRESS Peer Review of Electronic Search Strategies: 2015 Guideline Statement. J Clin Epidemiol 2016;75:40-6.

16 Veritas Health Innovation. Covidence systematic review software. Melbourne, Australia. Available: www.covidence.org

17 Patton MQ. Qualitative research and evaluation methods. 4 ed. Sage, 2014.

18 Boers M, Beaton DE, Shea BJ, et al. OMERACT filter 2.1: elaboration of the conceptual framework for outcome measurement in health intervention studies. J Rheumatol 2019;46:1021-7. 